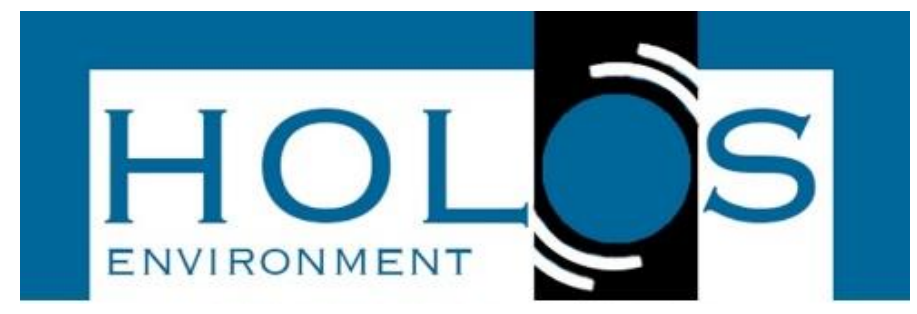

\title{
EMPREGO DE KRIGAGEM ORDINÁRIA PARA ESTIMAR A DISTRIBUIÇÃO ESPACIAL DE LITOFÁCIES PERMEÁVEIS: EXEMPLO DO AQUÍFERIO RIO CLARO EM PAULÍNIA/SP
}

\section{USE OF ORDINARY KRIGING TO ESTIMATE THE DISTRIBUTION OF THE PERMEABLE LITHOFACIES: EXAMPLE OF RIO CLARO AQUIFER IN PAULÍNIA/SP}

\author{
Elias Hideo Teramoto'; Hung Kiang Chang ${ }^{1,2}$
}

Artigo recebido em: 07/06/2018 e aceito para publicação em: 11/12/2018. DOI: http://dx.doi.org/10.14295/holos.v18i2.12297

Resumo: A distribuição de valores de condutividade hidráulica representa uma informação crucial para elaboração de modelos matemáticos de fluxo e transporte de contaminantes, bem como para o planejamento e implantação de sistemas de remediação para remoção de contaminantes em subsuperfície. É prática comum a construção dos campos de condutividade hidráulica de informações discretas, obtidas a partir da construção de modelos geológicos. Contudo, tal abordagem apresenta como limitação a subjetividade na elaboração de tais modelos, uma vez que a adoção de critérios para definição da distribuição de fácies e contatos litológicos envolve algum grau de subjetividade. No presente trabalho é proposta uma abordagem distinta, em que informações geológicas de subsuperfície são convertidas em valores de condutividade hidráulica. Estes valores são empregados como dados de entrada para construção de campos tridimensionais de condutividade hidráulica, utilizando o método geoestatístico Krigagem ordinária. A distribuição dos valores de condutividade hidráulica acima de um ponto de corte indica a geometria e a conectividade das fácies permeáveis. Esta abordagem foi testada no Aquífero Rio Claro, em Paulínia (SP), a partir de dados geológicos de subsuperfície coletados em 255 pontos. Os resultados indicam que os valores elevados de condutividade hidráulica se distribuem ao longo de uma faixa contínua, de direção leste-oeste, e refletem o contexto deposicional do Aquífero Rio Claro na área. A geometria dos corpos arenosos tem importantes implicações para determinação da arquitetura de fácies da Formação Rio Claro no munícipio de Paulínia e, de maneira similar, no comportamento do fluxo de água subterrânea e transporte de contaminantes no aquífero raso local.

Palavras-chave: Krigagem. Geoestatística. Condutividade hidráulica. Aquífero Rio Claro. Heterogeneidade geológica.

\footnotetext{
Abstract: The distribution of hydraulic conductivity values represents crucial information for the elaboration of mathematical models of flow and transport of contaminants, as well as the planning of the remediation systems to contaminant removal in the subsurface. It is common practice to construct the hydraulic conductivity fields from discrete information obtained from the construction of geological models. However, such an approach presents as a limitation the subjectivity in the elaboration of such models, since this stage involves subjectivity in the adoption of criteria used in the definition of facies distribution and lithological contacts. In the present work a different approach is proposed, in which geological information of sub-surfaces are converted to values of hydraulic conductivity, which are used as input

${ }^{1}$ Laboratório de Estudos de Bacias (LEBAC), UNESP - Campus de Rio Claro, SP. Centro de Estudos Ambientais (CEA), UNESP - Campus de Rio Claro, SP. E-mails: (teramoto@rc.unesp.br, chang@rc.unesp.br)

2 Departamento de Geologia Aplicada (DGA), UNESP - Campus de Rio Claro, SP.
} 
data for the construction of three-dimensional hydraulic conductivity fields from ordinary geostatistical methods. The distribution of hydraulic conductivity values above a cutoff value indicates the geometry and connectivity of permeable facies. The proposed approach was tested in the Rio Claro Aquifer in Paulínia/SP from the use of sub-surface geological data from 255 borehole data. The results indicate that the high values of hydraulic conductivity are distributed along a continuous belt of east-west direction and reflect the depositional context of the Rio Claro Aquifer, in which the sandy facies are the result of the amalgamation of channel fills and marginal bar, with marked vertical accretion along a narrow belt. The sandy bodies geometry has important implications for the determination of the facies architecture of the Rio Claro Formation in the municipality of Paulínia as well as the behavior of the groundwater flow and contaminants transport in the local shallow aquifer.

Palavras-chave: Kriging. Geostatistic.Hydraulic conductivity. Rio Claro Aquifer. Geological heterogeneities.

\section{INTRODUÇÃO}

A condutividade hidráulica (K) é uma propriedade dos aquíferos que descreve a facilidade com que um fluido pode se mover através de poros, sendo dependente da permeabilidade intrínseca do aquífero e da densidade e viscosidade do fluido. As variações da condutividade hidráulica nos aquíferos estão intimamente relacionadas às variações litológicas de seu arcabouço.

Diversos trabalhos, destacando-se Fogg (1986), Kolterman \& Gorelock (1996), Maxwell et al. (2008), Bianchi et al. (2011), Zhang et al. (2013), dentre outros, têm argumentado que em meios heterogêneos o aspecto mais importante para o fluxo e transporte de solutos é dado pela continuidade e interconectividade das fácies permeáveis, em oposição à visão tradicional de que estes fenômenos são estritamente controlados pela distribuição randômica da condutividade hidráulica. Fogg (1986) verificou que em aquíferos de origem fluvial, com fácies arenosas de canal esparsas e desconectadas, as velocidades de fluxo e transporte de solutos são 10 a 1000 vezes menores do que em porções do aquífero onde estas fácies são bem interconectadas. A geometria e a distribuição de corpos arenosos interconectados e de elevada permeabilidade podem ter grande impacto no transporte de contaminantes, fornecendo vias preferenciais para uma migração rápida e gerando o efeito conhecido por "canalização" de contaminantes, descrito por Moreno \& Tsang (1994), Fogg (1996), Bianchi et al. (2011), Zhang et al. (2013), dentre outros.

As técnicas geoestatísticas, representam importantes ferramentas para estimativas de condutividade hidráulica em locais não amostrados. Conceitualmente apresentado por Matheron (1960), a krigagem representa um método de estimativa no qual os valores interpolados são modelados por uma função ponderadora que estabelece 
a interdependência espacial entre os dados analisados, fornecendo a melhor predição linear e não-tendenciosa. A aplicação da krigagem para avaliar a distribuição espacial dos valores de condutividade hidráulica em aquíferos heterogêneos foi demonstrada em muitos trabalhos, incluindo Rehfeldt et al. (1992), Eggleston et al. (1996), Patriarche et al. (2005), Simo et al. (2013).

Regionalmente, o Aquífero Rio Claro é representado por litologias diversas (arenitos limpos a argilosos, arenitos microconglomeráticos, argilitos arenosos e siltitos argilosos), depositadas em um ambiente fluvial meandrante (FERNANDES, 1997). Este contexto deposicional é responsável pela forte heterogeneidade do aquífero em escala local (TERAMOTO, CHANG \& CAETANO-CHANG, 2017), fato que contribui para a existência de elevado grau de incerteza quanto à distribuição espacial das propriedades do aquífero e, por consequência, na ineficiência dos sistemas de remediação de áreas contaminadas por hidrocarbonetos aí implantados. Por esta razão, o presente trabalho empregou técnicas geoestatísticas para avaliar a distribuição dos valores de $\mathrm{K}$, particularmente a continuidade das litofácies dotadas de elevados valores de permeáveis, representado pelas fácies arenosas que correspondem a antigos depósitos de preenchimento de canal/barra marginal.

\section{MATERIAIS E MÉTODOS}

\subsection{Localização da área de Estudo e Geologia Regional}

A área de estudo situa-se no munícipio de Paulínia (Figura 1), porção centroleste do Estado de São Paulo. Na região de Paulínia afloram rochas do Embasamento Cristalino pré-cambriano e unidades litoestratigráficas da Bacia do Paraná - Subgrupo Itararé (Carbonífero superior ao Permiano inferior), rochas intrusivas básicas da Formação Serra Geral (Cretáceo inferior) e depósitos cenozoicos atribuídos à Formação Rio Claro por Melo (1995) e Fernandes (1997) - além de depósitos quaternários (Figura 1). A espessura do pacote de rochas sedimentares (Subgrupo Itararé, Formação Rio Claro e depósitos quaternários) sobre o substrato pré-cambriano é relativamente delgada nessa região, atingindo em média pouco mais de uma centena de metros. 
Figura 1 - Mapa geológico da região de Paulínia e adjacências

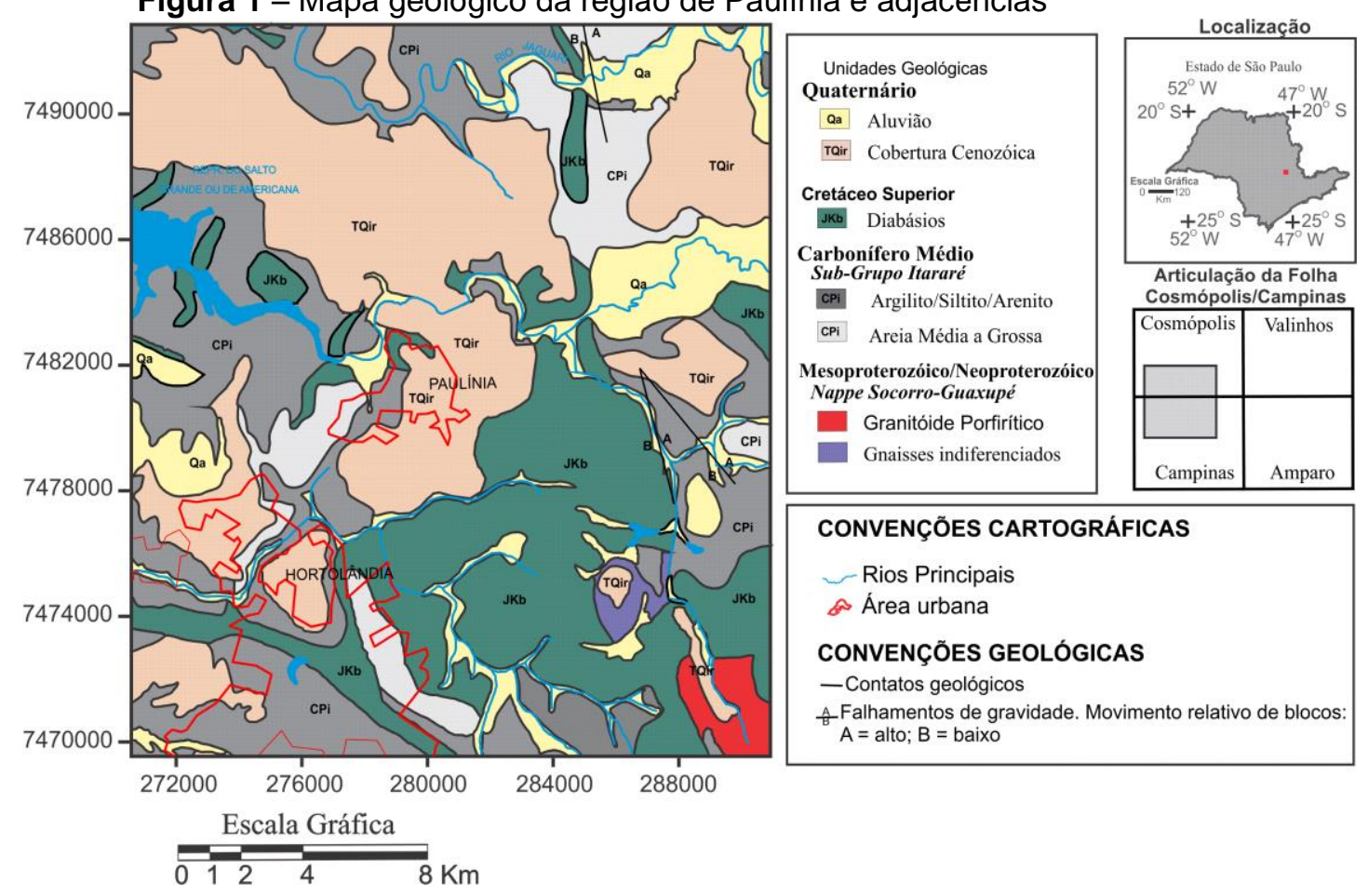

Fonte: Modificado de Fernandes(1997)

\subsection{Informações geológicas de subsuperfície}

As informações de subsuperfície empregadas neste trabalho foram adquiridas a partir de sondagens do tipo SPT (Standard Penetration Test), levantadas junto ao acervo das empresas onde estes ensaios foram realizados, e a partir da amostragem contínua pela técnica de direct push, executadas durante várias campanhas de investigação ambiental conduzidas na área. Um total de 255 pontos (Figura 2) com informações geológicas de subsuperfície foram utilizados na caracterização geológica local. 
Figura 2 - Distribuição dos 255 pontos com informações geológicas de subsuperfície empregados neste estudo

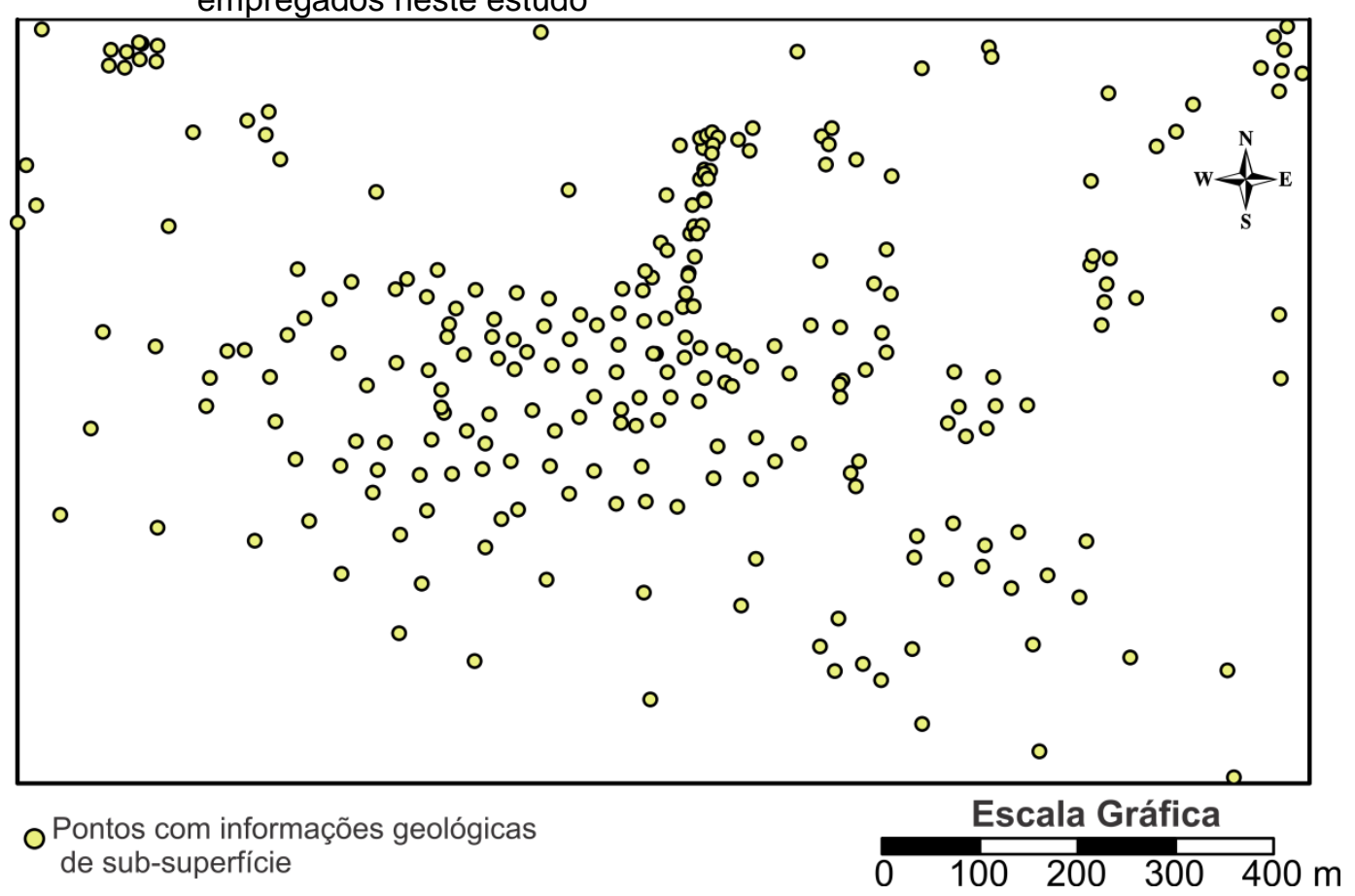

Os valores de condutividade hidráulica utilizados foram compilados por Teramoto, Chang \& Caetano-Chang (2017), a partir da consulta a diversos trabalhos. Segundo esses autores, os dados obtidos a partir dos ensaios de slug foram interpretados empregando-se o método proposto por Hvorslev (1951).

Nos poços de monitoramento foram instalados filtros em intervalos específicos, o que permitiu a determinação de condutividade hidráulica de cada uma das litologias identificadas.

\subsection{Análise Geoestatística}

Semivariogramas são usados para medir a correlação espacial e calcular a meIhor estimativa linear, sem viés, em locais onde não há medições disponíveis. A análise dos semivariogramas pode revelar a existência ou não de anisotropia na estruturação espacial da variável analisada e, se esta existir, identificar as duas direções principais da anisotropia. A relação entre semivariância e distância entre os pares de amostras é dada pela fórmula:

$$
\gamma(h, \theta)=\frac{1}{2 N(h, \theta)} \sum_{j=1}^{N(h, \theta)}\left[Z\left(x_{j}\right)-Z\left(x_{j}+h\right)\right]^{2}
$$


Onde $Z\left(x_{j}\right)$ e $Z\left(x_{j}+h\right)$ representam variáveis randômicas nos pontos $x_{j}$ e $x_{j}$; $h$ representa a distância entre as amostras; $\theta$ representa a direção entre dois pares de pontos; $\gamma(h)$ representa o valor estimado de semivarância para o lag $h ; N(h, \theta)$ é o número de pares em determinada distância h e ângulo $\theta$.

A ideia central da krigagem é estimar o valor de um atributo em um determinado ponto, a partir do cálculo da média ponderada dos valores conhecidos da função na vizinhança do ponto, onde o peso de cada dado está relacionado à correlação espacial previamente estabelecida através do modelo variográfico. A estimativa fornecida da variável de interesse no ponto $x_{0}$ é dada por:

$$
\hat{Z}\left(x_{0}\right)=\sum_{i=1}^{n} w_{i}\left(x_{0}\right) \cdot Z\left(x_{i}\right)
$$

A krigagem ordinária foi aplicada para estimativas da distribuição de In(K), empregando o aplicativo SGEMS (WU, BOUCHER \& ZHANG, 2008) em uma área de $1200 \mathrm{~m}$ de comprimento por $800 \mathrm{~m}$ de largura e $26 \mathrm{~m}$ de profundidade, em uma malha com 24.960 .000 pontos.

\section{RESULTADOS}

A partir dos perfis descritivos de poços de monitoramento, Teramoto (2015) estabeleceu os valores de condutividade hidráulica para cada litofácies identificada (Tabela 1).

Tabela 1 -Valores de condutividade hidráulica obtidos a partir de 116 poços de monitoramento, das diferentes litologias presentes na área de estudo (TERAMOTO, 2015)

\begin{tabular}{|c|c|c|c|c|}
\hline \multirow[b]{2}{*}{ Litologias } & \multicolumn{4}{|c|}{ Condutividade Hidráulica (m/s) } \\
\hline & Valor mínimo & Valor médio & Valor máximo & $\begin{array}{l}\text { № de } \\
\text { Ensaios }\end{array}$ \\
\hline Siltito argiloso & $7,39 \times 10^{-8}$ & $1_{10^{-6}}^{2,61 x}$ & $1_{10^{-6}}^{6,64 x}$ & 57 \\
\hline Argilito arenoso & $7,20 \times 10^{-6}$ & $10^{-5}{ }^{1,91 x}$ & $10^{-5} \begin{array}{l}3,82 x \\
-\end{array}$ & 31 \\
\hline Arenito médio argiloso & $5,23 \times 10^{-5}$ & $10^{-5}=$ & $10^{-5}{ }^{8,98 x}$ & 5 \\
\hline $\begin{array}{l}\text { Arenito médio a grosso } \\
\text { microconglomerático }\end{array}$ & $1,03 \times 10^{-4}$ & $10^{-4,36 x}$ & $10^{-4}$ & 23 \\
\hline
\end{tabular}

Verifica-se que cada grupo litológico apresenta baixa variabilidade nos valores de condutividade hidráulica, com exceção dos siltitos argilosos que ostentam variados graus de faturamento. A presença dessas fraturas é responsável por conferir valores 
de condutividade hidráulica muito superiores aos esperados para essa litologia, sendo possível afirmar que a variabilidade no grau de fraturamento desse litotipo é responsável pela ampla faixa de variação dos valores de $\mathrm{K}$, que alcança duas ordens de grandeza.

Esses valores de condutividade hidráulica, obtidos em campo, abrangem várias ordens de grandeza. Para promover a linearização, foi efetuado o cálculo do logaritmo neperiano (In) desses valores. . Deste modo, os valores a serem interpolados pelas técnicas geoestatísticas são representados pelos valores de $\ln (\mathrm{K})$. A partir da associação entre a litologias e valores médios de condutividade hidráulica (Tabela 1), compôs-se uma tabela contendo as coordenadas $(x, y)$ de cada ponto de sondagem SPT (Figura 2), as respectivas cotas altimétricas (z) e $\ln (\mathrm{k})$ (Figura 3).

Figura 3 - Transformação de informações geológicas de subsuperfície em dados tridimensionais de condutividade hidráulica

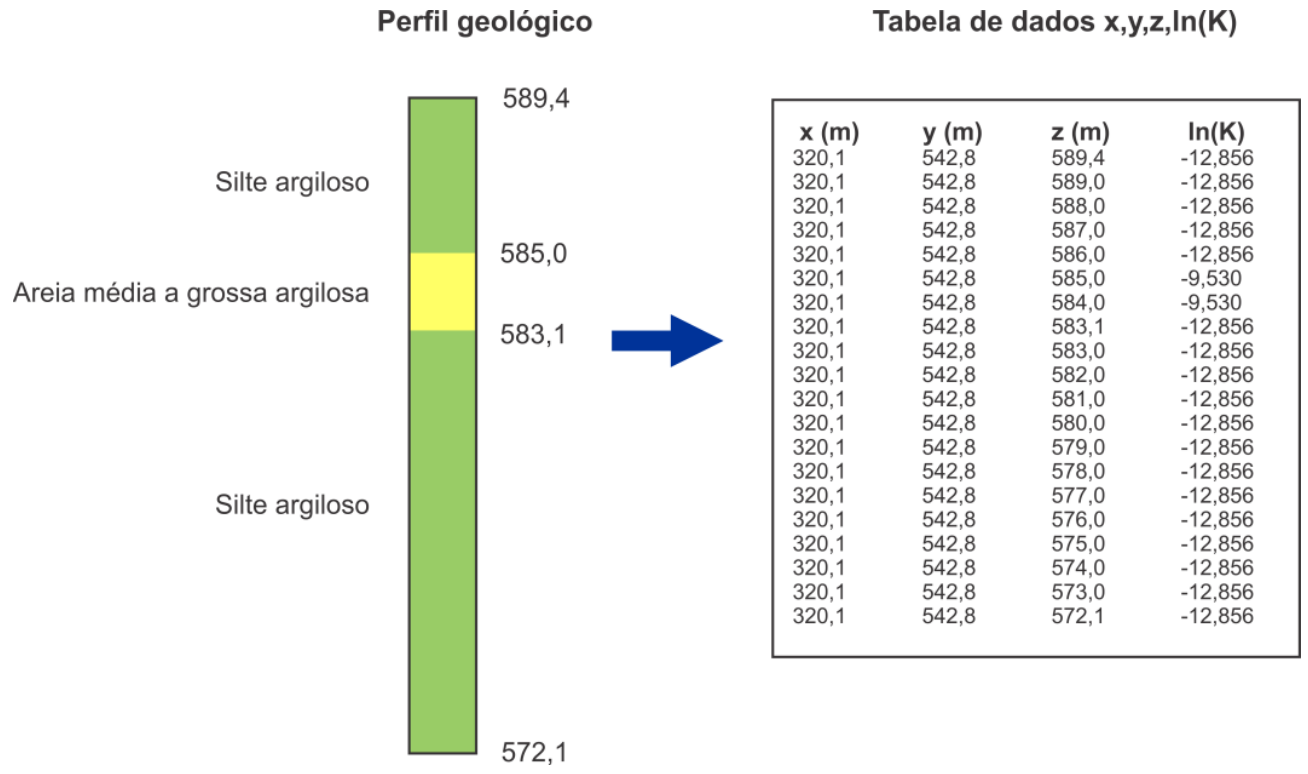

Tendo em vista que os valores médios de condutividade hidráulica abrangem intervalos de quatro ordens de grandeza (Tabela 1), a análise exploratória do conjunto de dados disponíveis foi mediada pela análise estatística paramétrica, cujos resultados são apresentados na Tabela 2. 
Tabela 2 - Resultados da análise estatística paramétrica dos valores de $\ln (\mathrm{K})$, obtidos a partir da transformação de informações litológicas

\begin{tabular}{cc}
\hline Parâmetro & Valor \\
\hline$N^{\circ}$ de amostras & 4641 \\
Mínimo & $-12,856$ \\
Máximo & $-8,352$ \\
Média & $-11,441$ \\
Erro padrão & 0,025 \\
Variância & 2,926 \\
Desvio-padrão & 1,711 \\
Mediana & $-12,856$ \\
Simetria & 0,567 \\
Curtose & 152,828 \\
Coeficiente de variação & $-14,951$ \\
\hline
\end{tabular}

O histograma dos valores de $\ln (\mathrm{K})$ apresenta distribuição lognormal (Figura 4), com amplo predomínio de valores $-12,856$, em razão do franco predomínio da litologia silte argiloso, de fácies de planície de inundação, que compõe grande parte do arcabouço do Aquífero Rio Claro na área de estudo.

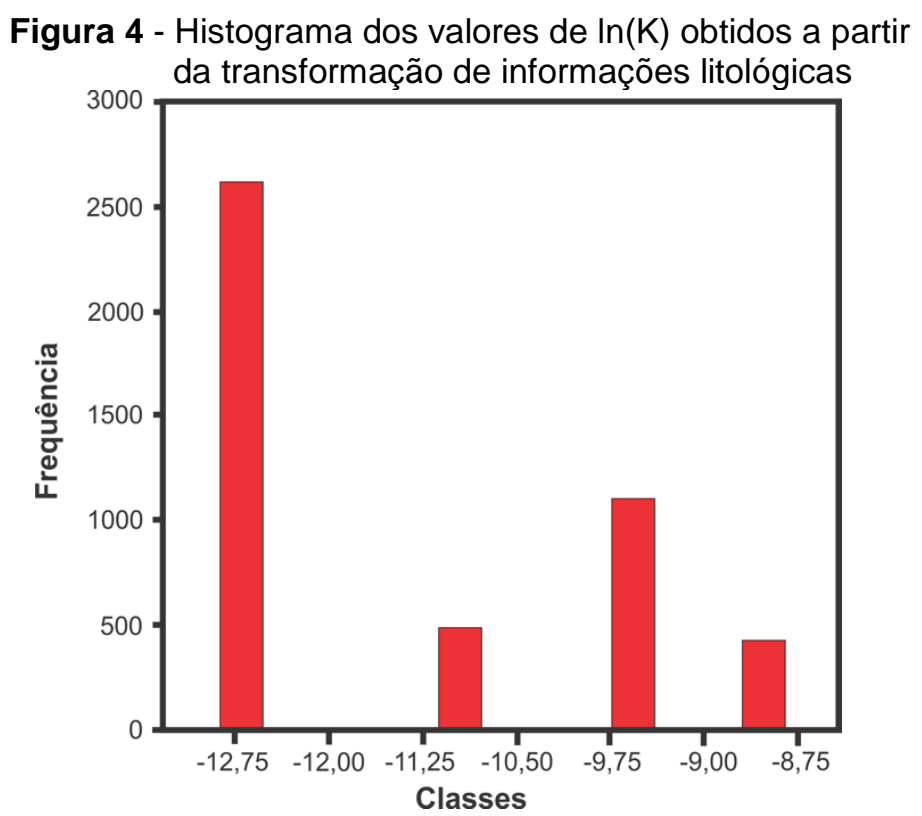

A Figura 5 ilustra a distribuição tridimensional das informações geológicas disponíveis, em valores equivalentes de $\ln (K)$, com base na correspondência entre litologia e valor médio de $\mathrm{K}$ (Tabela 1 ). 
Figura 5 - Distribuição tridimensional dos valores de $\mathrm{K}$ nas sondagens da área

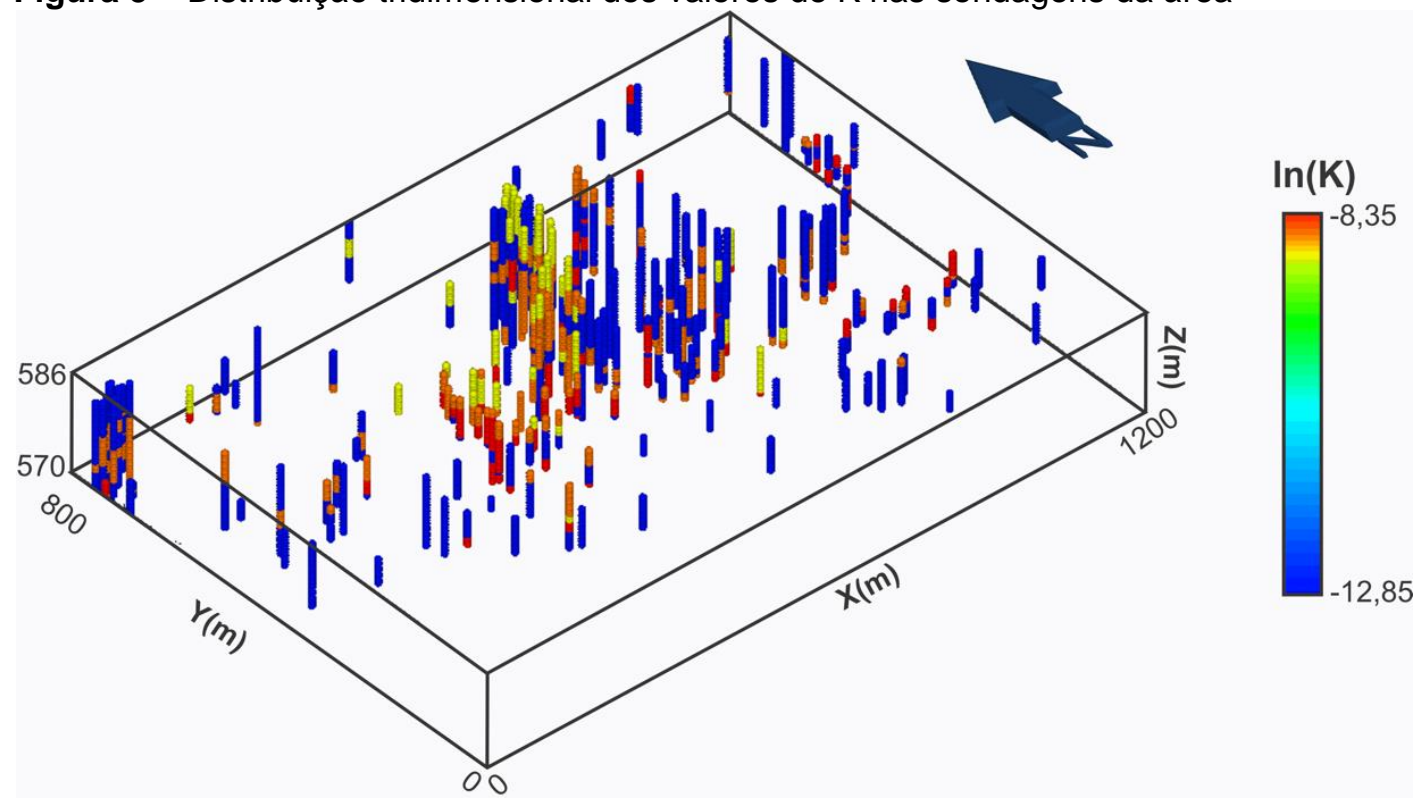

O semivariograma experimental (Figura 6) foi calculado com espaçamento de $40 \mathrm{~m}$ e ajustado com um modelo variográfico esférico, com soleira igual a 2,3 e efeito pepita igual a 1,0. Na direção leste-oeste (Figura 6a), a amplitude modelada foi de 196 m, enquanto na direção norte-sul (Figura 6b), a amplitude modelada foi de $144 \mathrm{~m}$, revelando a existência de anisotropia geométrica.

Figura 6 - Variograma experimental e modelado: a) direção leste-oeste; b) direção norte-sul
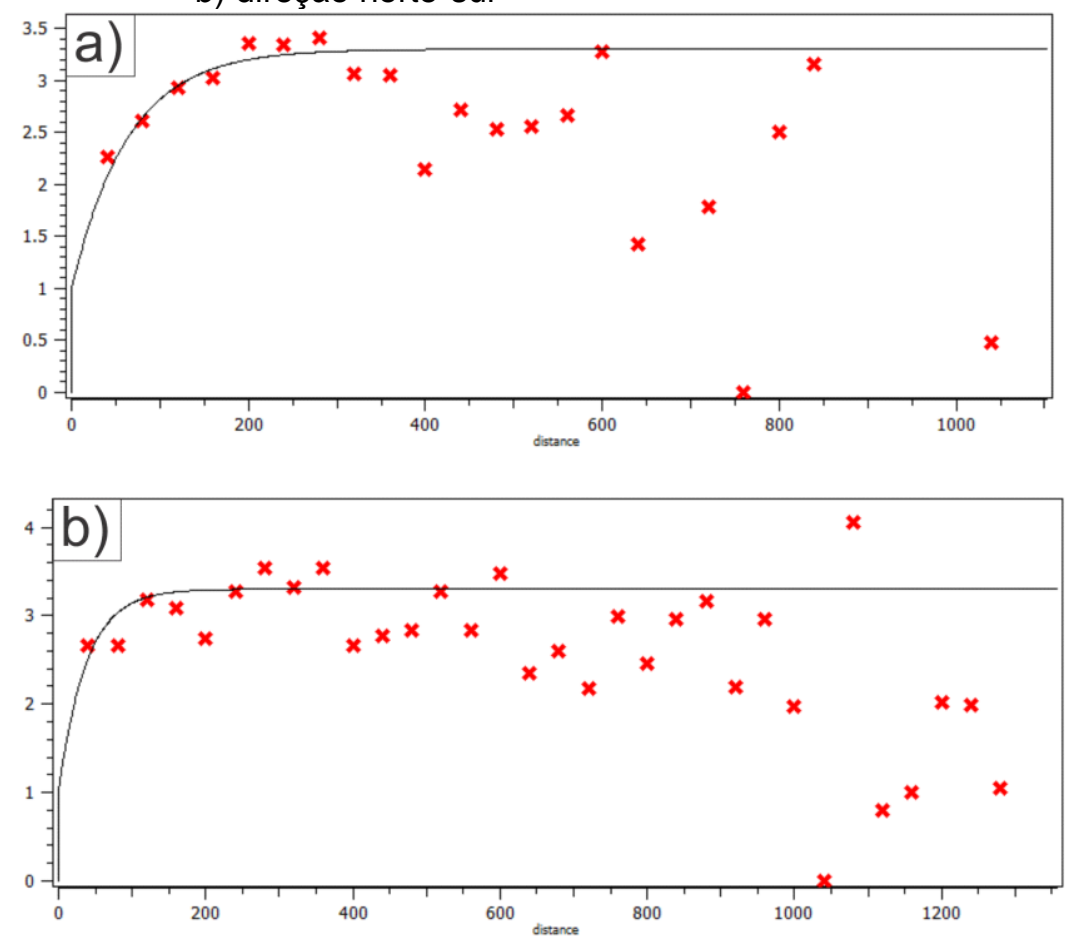
Aos dados do modelo variográfico ajustado (Figura 6) foi empregada a krigagem ordinária tridimensional para gerar os campos de condutividade hidráulica, utilizando-se o aplicativo SGEMS. Neste trabalho buscou-se avaliar a distribuição das zonas mais permeáveis do aquífero, com valores superiores de -9,90 (correspondente a valor de $\mathrm{K}=5,0 \times 10^{-5} \mathrm{~m} \cdot \mathrm{s}^{-1}$ ). Por esta razão, os campos tridimensionais de $\mathrm{K}$, gerados pela krigagem ordinária, foram inseridos no aplicativo Earth Volumetric Studio (EVS) (C-TECH, 2016) e configurados para representação gráfica apenas de valores de $\ln (K)$ superiores a $-9,90$, suprimindo-se valores inferiores. Na Figura 7 são representadas as porções com valores elevados de $\mathrm{K}$, enquanto as áreas vazias representam valores inferiores de $\mathrm{K}$. As porções com valores de condutividade superiores a 9,90 estão associadas à distribuição de litologias arenosas e se dispõem ao longo de uma faixa contínua, com direção aproximada leste-oeste.

Figura 7 - Porções com valores de $\ln (\mathrm{K})$ acima de $-9,90\left(\mathrm{~K}=5,5 \times 10^{-5} \mathrm{~m} \cdot \mathrm{s}^{-1}\right)$

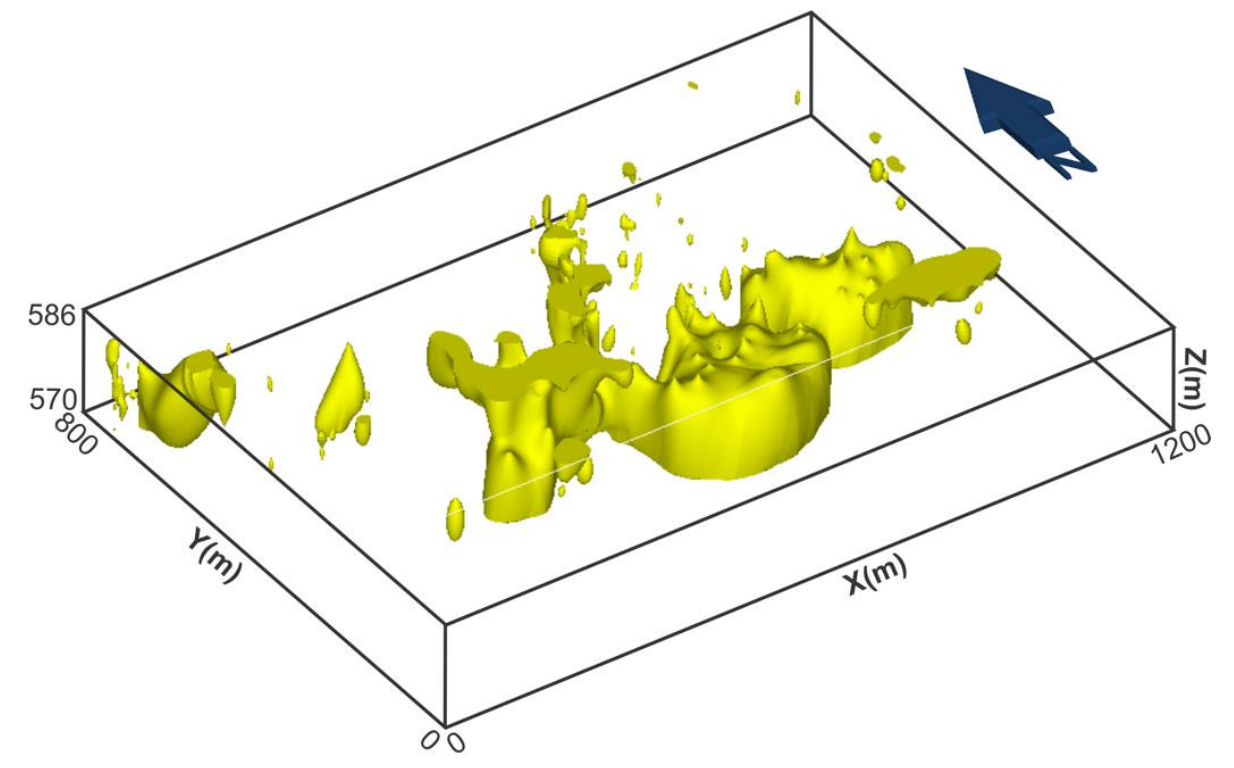

\section{DISCUSSÃO}

A construção dos campos de condutividade hidráulica constitui informação crucial para conformação de modelos matemáticos de fluxo e previsão de transporte de solutos. De maneira similar, permite prever a eficiência dos sistemas de remediação baseada na extração multifásica ou injeção de oxidantes, uma vez que o sucesso da remediação está diretamente atrelado à distribuição dos valores de permeabilidade. 
A abordagem mais comumente empregada consiste na construção de modelos geológicos tridimensionais, com a distribuição da condutividade hidráulica atrelada à distribuição litológica nesses modelos. Tal abordagem, contudo, é sujeita à subjetividade na interpretação dos dados geológicos e nos critérios utilizados para determinação dos contatos entre as litofácies formadoras do aquífero. A premissa empregada assume que a conversão de informações geológicas (de natureza qualitativa) em valores de condutividade hidráulica (um atributo quantitativo) permite estimar confiavelmente a geometria das litologias com elevados valores de permeabilidade, por intermédio de técnicas geoestatísticas. A distribuição lateral, continuidade e anisotropia refletem a arquitetura de fácies sedimentares. Cabe destacar que a análise variográfica, precedendo a interporlação por krigagem, permite avaliar a variabilidade e correlação espacial dos valores de $\mathrm{K} \mathrm{e}$, assim, inferir a geometria e continuidade lateral das litofácies arenosas no aquífero.

O campo de condutividade hidráulica gerado pela krigagem ordinária indica que os maiores valores de condutividade hidráulica se distribuem ao longo de um faixa de direção aproximada leste-oeste e refletem a geometria e distribuição litotipos arenosos (fácies de canal) na área. O predomínio de valores reduzidos de condutividade hidráulica é condizente com a ampla distribuição de sedimentos pelíticos, de fácies de planície de inundação, na Formação Rio Claro na região de Paulínia, conforme atestado por Teramoto, Chang e Caetano-Chang (2017).

A continuidade lateral das fácies com elevada permeabilidade aqui identificadas apresenta corpos arenosos com geometrias distintas daquelas observadas nos resultados do Teramoto, Chang e Caetano-Chang (2017) que empregaram o método estocástico das Cadeias de Markov para gerar diferentes cenários geológicos. O maior grau de conectividade observada no presente trabalho (Figura 7 ) em oposição aos observados por do Teramoto, Chang e Caetano-Chang (2017) se deve ao fato da interpolação por krigagem suavizar as diferenças entre dois pontos de observação, gerando uma distribuição gradativa que confere uma maior continuidade lateral. De maneira oposta, as Cadeias de Markov representam mais realisticamente os contatos laterais entre as diferentes fácies, que truncados por contatos abruptos característicos de contatos erosivos. É provável que a distribuição espacial das fácies arenosas apresentadas no presente trabalho esteja mais próximas das condições reais do aquífero, mas as dimensões mais realistas destas fácies sejam aquelas observadas no trabalho de Teramoto, Chang e Caetano-Chang (2017) 
Tendo em vista que no munícipio de Paulínia se situa um importante pólo petroquímico nacional, o vazamento acidental de tanques de armazenamento de combustíveis é um cenário comum de contaminação em subsuperfície. A distribuição do LNAPL (Light Non-Aqueous Liquid Phase) é fortemente dependente das variações geológicas, como demonstrado por Jeong \& Chaberneau (2014), e as constatações do presente trabalho podem auxiliar no entendimento no comportamento do LNAPL em subsuperfície. Regiões onde se verifica maior continuidade das fácies de elevada permeabilidade, constituem porções onde o movimento advectivo dos contaminantes na água subterrânea é controlado pelo efeito de canalização, que tem fortes implicações no entendimento do comportamento das plumas de fase dissolvida.

É relevante mencionar que a premissa da abordagem proposta no presente trabalho assume que a média geométrica representa o valor mais representativo de condutividade hidráulica nas litofácies. Entretanto, a despeito desta premissa, existem variações dos valores de condutividade hidráulica dentro de cada litofácies e estas variações representam importantes fontes de incertezas. Para exemplificar este problema, a faixa de variações dentro da litofácies silte argiloso alcança uma ordem de grandeza (Tabela 1) e é provável que o contraste da condutividade hidráulica dentro do aquífero seja maior que o estimado no presente trabalho, com efeito pronunciado em modelos matemáticos de transporte de solutos. Outro fator a ser considerado é que a krigagem promove a suavização dos valores de condutividade hidráulica, o que pode promover desvios significativos, uma vez que a transição no contato erosivo entre as fácies gera mudanças abruptas de condutividade hidráulica.

\section{CONCLUSÕES}

O emprego de métodos geoestatísticos a um conjunto de dados de valores médios de condutividade hidráulica, obtidos a partir de descrições geológicas de subsuperficie, mostrou-se eficiente para inferir a distribuição hidrofaciológica permeável do Aquífero Rio Claro. Verificou-se que as fácies mais permeáveis se distribuem em uma faixa leste-oeste na área, coincidindo com a distribuição dos litotipos arenosos, em um contexto deposicional dominado por amalgamento de canais. As fácies arenosas estão embutidas em depósitos dominados por fácies pelíticas de planície de inundação, com baixos valores de condutividade hidráulica. Os resultados permitiram apri- 
morar o entendimento da distribuição dos valores de condutividade hidráulica no Aquífero Rio Claro e as implicações desta distribuição no regime de fluxo de água subterrânea e no entendimento da migração de contaminantes em subsuperfície.

\section{REFERÊNCIAS}

BIANCHI, Marco et al. Spatial connectivity in a highly heterogeneous aquifer: From cores to preferential flow paths. Water Resources Research, v. 47, n. 5, 2011.

C-Tech Development Corporation. The Earth Volumetric Studio. C-tech Development Corporation, Las Vegas, NV. 2016.

EGGLESTON, J. R.; ROJSTACZER, S. A.; PEIRCE, J. J. Identification of hydraulic conductivity structure in sand and gravel aquifers: Cape Cod data set. Water Resources Research, v. 32, n. 5, p. 1209-1222, 1996.

FERNANDES, A.J. Tectônica Cenozóica na Porção Media da Bacia do rio Piracicaba e sua Aplicação à Hidrogeologia, Tese (Doutoramento em Recursos Minerais e Hidrogeologia). Instituto de Geociências, Universidade de São Paulo. São Paulo, 244 F, 1997.

FOGG, G.E. Groundwater flow and sand body interconnectedness in a thick, multiple-aquifer system. Water Resources Research, v. 22, n. 5, p. 679-694, 1986.

HVORSLEV, M.J. Time Lag and Soil Permeability in Ground-Water Observations, Bull. 36, Waterways Exper. Sta. Corps of Engrs, U.S. Army, Vicksburg, Mississippi, p. 1-50. 1951.

JEONG, Jaehak; CHARBENEAU, Randall J. An analytical model for predicting LNAPL distribution and recovery from multi-layered soils. Journal of contaminant hydrology, v. 156, p. 52-61, 2014.

KOLTERMANN, C.E.; GORELICK, S.M. Heterogeneity in sedimentary deposits: A review of structure-imitating, process-imitating, and descriptive approaches. Water Resources Research, v. 32, n. 9, p. 2617-2658, 1996.

MATHERON, Georges. Traité de géostatistique appliquée. 1 (1962). Editions Technip, 1962.ou 1960?

MAXWELL, R.M.; CARLE, S.F.; TOMPSON, A.F.B. Contamination, risk, and heterogeneity: on the effectiveness of aquifer remediation. Environmental Geology, v. 54, n. 8, p. 17711786, 2008.

MELO, M.S. A Formação Rio Claro e depósitos associados: sedimentação neocenozóica na Depressão Periférica Paulista, São Paulo. Tese (Doutorado) - Instituto de Geociências, Universidade de São Paulo. São Paulo, 144F, 1995.

PATRIARCHE, Delphine; CASTRO, Maria Clara; GOOVAERTS, Pierre. Estimating regional hydraulic conductivity fields-A comparative study of geostatistical methods. Mathematical Geology, v. 37, n. 6, p. 587-613, 2005. 
REHFELDT, Kenneth R.; BOGGS, J. Mark; GELHAR, Lynn W. Field study of dispersion in a heterogeneous aquifer: 3. Geostatistical analysis of hydraulic conductivity. Water Resources Research, v. 28, n. 12, p. 3309-3324, 1992.

SIMO, Aubin Thibaut Guekie et al. Reconstructing hydraulic conductivity field for hydrogeological modeling in an urban environment. Engineering Geology, v. 158, p. 119-134, 2013.

TERAMOTO, E.H.; CHANG, H.K. Caracterização hidrogeológica e simulação numérica em área do distrito industrial de Paulínia. Águas Subterrâneas. V. 22, N.1, p. 129-144. 2008.

TERAMOTO, E.H. Estudo da efetividade da atenuação natural de compostos BTEX em uma área contaminada por querosene de aviação. Tese de Doutorado (Programa de Pós-Graduação em Geociências, Área de Concentração em Geociências e Meio Ambiente). Instituto de Geociências e Ciências Exatas, Universidade Estadual Paulista, Rio Claro, SP. 238 f. 2015.

TERAMOTO, E.H.; CHANG, H. K.; CAETANO-CHANG, M.R. Transporte de solutos em diferentes cenários geológicos gerados por modelos estocásticos de cadeias de Markov. Águas Subterrâneas, v. 31, n. 4, p. 316-326, 2017.

WU, Jianbing; BOUCHER, Alexandre; ZHANG, Tuanfeng. A SGeMS code for pattern simulation of continuous and categorical variables: FILTERSIM. Computers \& Geosciences, v. 34, n. 12, p. 1863-1876, 2008.

ZHANG, Y.; GREEN, C. T.; FOGG, G. E. The impact of medium architecture of alluvial settings on non-Fickian transport. Advances in Water Resources, v. 54, p. 78-99, 2013. 